\title{
Artificial Intelligence in Pharma
}

\author{
Babu Keziah $A^{1}$, Shirlin $M S^{2}$; Devi Manjula $A S^{3}$
}

\author{
${ }^{1,2,3}$ Department of Pharmacy Practice, College of Pharmacy- Sri Ramakrishna Institute of \\ Paramedical Sciences, Coimbatore, 641044, India
}

\begin{abstract}
This paper aims to provide a better understanding of the possible applications of Artificial Intelligence (AI) in the field of Pharmacy. Artificial intelligence basically involves complex information processing. It enables machines to carry out certain functions which were initially done by human and which required human intellect. Over the past few years, the use of artificial intelligence in the pharma has grown unimaginably and is now able to revolutionize the way drugs are discovered and handled. Al can impact the field of Pharmacy at multiple levels. Pharmacy and medical education, drug development, personalization of drug therapy, drug safety, rational drug use, manufacture and formulation of dosage forms are areas of impact by Al. The fact that Al can displace humans at their tasks is a matter of concern. Although there are ethical concerns regarding the implementation of Al in healthcare, it can be assured that no machine or system can replace many of the humanitarian duties.
\end{abstract}

KEYWORDS: Artificial Intelligence; Drug discovery; Personalized medicine

\section{INTRODUCTION}

T The Artificial Intelligence (AI) is a field that deals with the design and application of algorithms for analyzing and interpreting information. (1) It is the use of advanced computer algorithms to perform tasks like humans, such as making observations and interpretations (2). It is based on an understanding of intelligent behaviour, and the creation of algorithms that exhibit such behaviour (3). What AI does is to identify potential problems and provide various options to solve them (1).

In AI, machines imitate critical thinking capabilities with other human personalities. (2) As of now, most of the applications of AI are primitive as they are designed to do limited tasks and to a limited extent. AI works through multiple paths, allowing systems to discover new patterns and derive their own rules when given with data and new experiences (4). Some of the critical applications of AI in pharma are designing treatment plans, checking the accuracy of medicine and in drug creation (5).

Major pharma companies are adopting AI software or partnering with AI-based startups to accelerate drug discovery. With AI, customization of dosages can be achieved based on individual patient characteristics. AI is also increasingly being used to detect drug effectiveness and to understand adverse drug events. It may also help accelerate drug approval processes by the regulatory authorities (6).

A number of AI-based tools have been developed such as IBM Watson for oncology, Robot pharmacy (for preparation and dispensing of medicines for individual patients), Erica robot (understands and answers questions with human-like facial expressions) etc (7). In India, the use of AI software for research and development has begun only over the recent years (6). Not one or two but several walks of the broad science of Pharmacy can benefit from the use of AI technology in the future.

Pharma education: Artificial intelligence

Correspondence: Keziah Ann Babu, College of Pharmacy, Sri Ramakrishna Institute of Paramedical Sciences,

Coimbatore, 641044, Tamil Nadu, India. E-mail: keziahannbabu@gmail.com

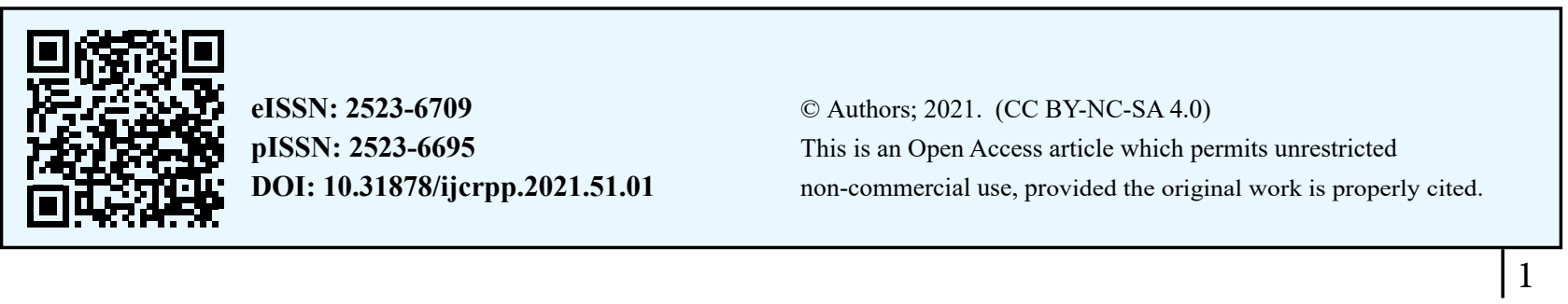


covers various areas in the health sciences. Computerization of pharma education by means of well-structured courses, enhanced by assessments and inputs, from the internet, social networks, and advanced platforms would help improve the standards of pharma education. It must include a feedback system that helps to improve the functioning and gives information on user satisfaction. Due to the extensive availability of the internet, it is easy to search for information in a fraction of seconds. There are several web pages available to guide people, pharmacists as well as other healthcare professionals on medical information (2).

Personalized medicine: Treatment is quickly shifting from traditional to patient-centred approach. This can be further successful by the integration of technologies such AI to suit the needs of personalized medicine, for progressing through medical examinations, preventive monitoring, drug dosage adjustment and customized treatment plans for the patient (6). In a few years from now, treatment regimens will be developed according to individual genomic traits. Such a strategy has already gained momentum in oncology. Platforms like Oncompass and Foundation Medicine provide suggestions to individual patients based on their genetic makeup. Artificial intelligence can be utilized in clinical decision making to check the patient's records, to recommend treatments and predict their potential outcomes (2).

Drug discovery: Artificial intelligence can be suited to analyze complex statistical data in medical research. The extent of clinical research is becoming vague as research becomes more dependent on large amounts of data processing. In such a scenario, pharmaceutical companies can benefit from AI. AI could help to extend the boundaries of clinical research by processing terabytes of data to find the perfect fit, thereby easing the time-consuming task for researchers. (8) Drug discovery and development is not easy, and it costs billions and is extensively timeconsuming. Numerous molecules could have the potential as leads for a large number of diseases. This is where AI can benefit pharmaceutical companies (7). By accelerating the development of new drugs, researchers can identify multiple drug candidates for clinical trials and can develop more and more models to study pharmacokinetics and pharmacodynamics. AI plus clinical thinking will enable healthcare professionals to ensure the validity of research findings in the future (8).

AI systems could also be valuable in clinical research to identify, match and recruit suitable patients to relevant studies (9). AI can fasten the process of drug discovery, reduce expenses involved in $R$ \& $D$, improve success rates in clinical trials and promote the development of superior medicines. It can be used to predict potential toxicity in clinical trials. AI is capable of transforming the future of medical care but still needs to evolve in order to achieve positive outcomes (10). AI can be used for new drug target identification and validation and to develop better drug formulations, diagnostics or biomarkers. It can be used for repurposing drugs to find newer indications for existing drugs or drug candidates, thereby accelerating clinical drug trials (5).

Organic synthesis and designing, scoring of synthetic complexes, automation of molecular design, predicting outcomes of organic reactions, computer-aided synthesis and retrosynthesis, predicting drug performance in vitro testing, discovering off label use, predicting toxicity prior to clinical trials outcomes, personalization of medicine could be the key areas for AI-based focus (6).

Rational use of drugs: One of the roles of a pharmacist is to make ceratin that the prescriptions received, are appropriately supplied with the right medications at the right dose and quantity and to check for drug interactions and/or adverse drug events, in case of multiple medications. AI robots are being used more and more to carry out these functions that were previously done by humans (7).

AI systems can help organize dosage forms, route of administration or treatment plans in a better way and provide guidance to physicians (5). Algorithms could be used to standardize prescriptions by checking their appropriateness with the latest guidelines by updating the operating system from time to time (8). Machines with AI incorporated can also be applied for predicting and analyzing the outcomes of the treatment plan for various diseases and disorders (9).

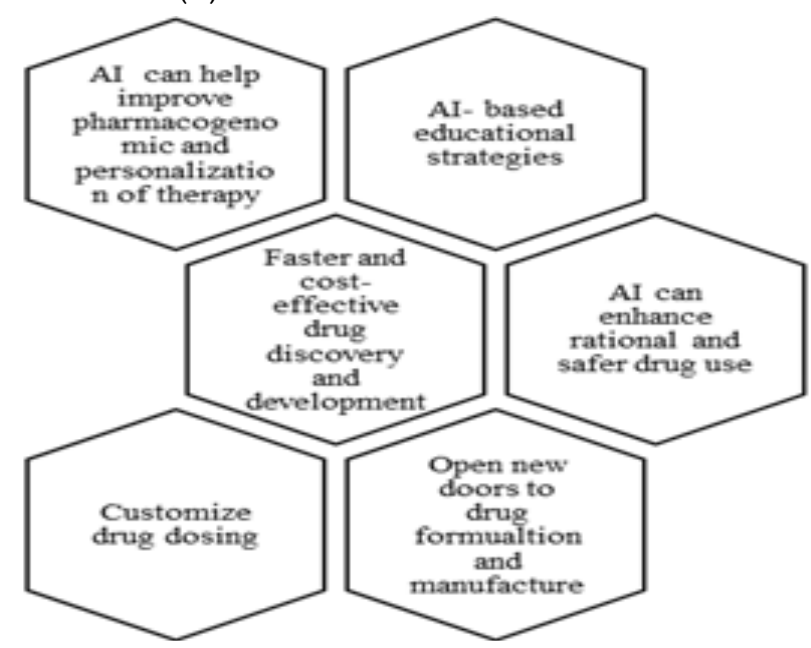

Figure.1. Applications of AI in Pharma; this figure is a representation of the possible applications of Artificial Intelligence in various walks of the field of Pharmacy.

Drug dosing: AI platforms that use patient's clinical data like drug doses, to create a customized treatment course are being developed. It can help create individual patient 
profiles to guide physicians in choosing the optimum drug dose plan (6).

Drug safety: Drug safety right from the stage of the lead compound to post- marketing is a key concern. Development of advanced AI tech to analyze the data can significantly lower the risk of drug-related toxicities. Artificial Intelligence in pharmacovigilance would help eliminate a huge load of manual work, thereby saving significant time and resources (6).

Manufacturing: When we talk about pharmaceuticals, drug manufacturing, beginning from design of dosage form to finished product, is very complex and requires close consideration of the raw materials and their processing factors. These factors can affect the quality of the finished product (1).

Over the past two decades, AI technology has been extensively used to develop and optimize complex formulations such as controlled release and immediate-release forms (11).

AI systems have the potential to reduce drug production time, improving safety and quality, and detect ways to repurpose existing drugs. The adoption of AI technologies could automate various processes such as drug logistics, tracking, packaging and processing and can reduce errors to a considerable extent (6).

\section{Conclusion}

AI will revolutionize the way drugs are manufactured, prescribed, and utilized. AI could help amplify several tasks in Pharmacy and overall medical care. It will also transform many aspects of the pharmaceutical industry and clinical pharmacotherapy but cannot assure to cure diseases and may replace humans for machines in the future. It cannot be expected to find solutions on a humanitarian basis. It can help healthcare workers to gain a better understanding of diseases, enable better clinical decision making and empower research.

\section{Source of Support: Nil}

Conflict of Interest: None Declared

\section{REFERENCES}

1. Sable $P$, Khanvikar VV, Sable $P$, Khanvilkar VV. Pharmaceutical Applications of Artificial Intelligence. Int $\mathrm{J}$ Pharma Res Heal Sci. 2018;6(2):2342-87.

2. Gawad J, Bonde C. Artificial Intelligence: Future of Medicine and Healthcare. Biochem Ind $\mathrm{J}$ 2017;11(2): 113 .

3. Ramesh AN, Kambhampati C, Monson JRT, Drew PJ. Artificial intelligence in medicine. Ann R Coll Surg Engl. 2004;86(5):334-8.

4. Ai WIS. Artificial intelligence ( AI ) in healthcare and research. Nuffield Council on Bioethics. May 2018:1-8.

5. Manikiran SS, Prasanthi NL. Artificial Intelligence:
Milestones and Role in Pharma and Healthcare Sector; Sector H. Pharma Times. 2019.

6. Use of Artificial Intelligence and Advanced Analytics in pharmaceuticals, FICCI. http://ficci. in/spdocument/23066/Knowledge-Paper-IndiaPharma-2019.pdf

7. Tomar B, Mishra V. Artificial Intelligence: The Beginning of a. 2018;12(2):72-6.

8. Artificial Intelligence in Healthcare, Academy of Royal Medical Colleges, 2019.

9. Jiang F, Jiang Y, Zhi H, Dong Y, Li H, Ma S, et al. Artificial intelligence in healthcare: Past, present and future. Stroke Vasc Neurol. 2017;2(4):230-43

10. Agrawal P. Artificial Intelligence in Drug Discovery and Development. J Pharmacovigil. 2018;6(2).

11. ĆJP. Artificial intelligence in pharma- ceutical product formulation: neural computing. 2009;15(4):227-36. 